\title{
A. THEORY
}

\section{PAPER I \\ MAGNETIG FIELDS IN ASTROPHYSIGS}

\author{
H. C. VAN DE HULST \\ University Observatory, Leiden, Netherlands
}

This symposium is held under the auspices of the International Astronomical Union at the department of electronics of a technical university. This combination of astronomy and technical electronics has already become so familiar, that it hardly strikes us as peculiar. This shows how strongly the case for electric and magnetic phenomena in a wide variety of astrophysical problems has been proven. However, all of this is comparatively recent history. One glance at an older epoch may illustrate this point and, perhaps, may help us to take fewer things for granted during our discussions.

Just fifty years ago, in 1906, Agnes Clerke [1] wrote a modern textbook, Problems in Astrophysics. In it the term magnetic field occurs only in one context. She devotes three pages to a problem that has an important place also in this symposium, namely the role of the sunspots in causing the terrestrial magnetic storms. Her discussion is concluded by the words: 'The machinery by which electromagnetic impulses are propagated from the sun to the earth, completely evades scrutiny. Sundry conjectures on the subject have been hazarded, but none of them rests on any sure basis. What we know about modes of communication is chiefly negative.' The discussions at this symposium will show to what extent the situation has improved.

\section{SOLAR PHENOMENA}

Two years after Miss Clerke's book, Hale discovered the magnetic fields of sunspots and again five years later, he believed he had found the general magnetic field of the sun. Knowledge about the sunspot fields has rapidly increased since that time, but the general field of the sun has been under constant debate. Only recent techniques allow the measurement of fields 
as low as a few gauss over the entire disc. Even then, caution is required in the interpretation.

Both types of investigations, of the spot-fields and of the general solar field, were prompted by superficial arguments: the apparent vortex structure in the $\mathrm{H} \alpha$ photographs in a spot-region and the striking pattern of the streamers on corona photographs, which look very much like magnetic lines of force. These arguments are suggestive but they ought to be justified by a later, more thorough investigation. If I am right, the precise interpretation of both phenomena is still discussed; we hope to hear more about them during this symposium. However, they form only a few of a multitude of phenomena known by the collective term solar activity.

Solar activity includes everything that is changing on the sun (with the traditional exception of the moving elements in the convective zone, the (consequent) granulation in the photosphere, and the (consequent) existence of a chromosphere with spicules). The observed changes included in the term solar activity have very different time scales. One extreme is the deep-seated cause of the 22-year cycle, which shows beautifully in the migration of the sunspot and prominence zones and in the reversal of dominant magnetic polarity at each half-cycle. The other extreme is formed by the storm bursts in the radio emission of the sun, which last for a fraction of a second. Between these extremes lie, for instance, the sunspots, which appear, develop and vanish in a month or so, and also the flares with all their associated effects that take about fifteen minutes.

It is not difficult to assign a rough order of cause and effect to these events. Roughly, the deep-seated and long-lived phenomena are cause, the others effect. If we wish to compare with the waves of the sea, the 22-year solar cycle corresponds to the fundamental tidal wave, and the solar radio bursts to the splashes and foam which we enjoy at the beach. It is, of course, an enormous task to track the chain of cause and effect in detail, but this is one of the jobs we are here for.

\section{HYDROMAGNETIGS}

I have not started, as Dr Bullard did on an earlier occasion [2] by giving a definition of magneto-hydrodynamics (which I shall call hydromagnetics): 'the study of the motion of fluids in the presence of magnetic fields'. The reason for mentioning this topic in the second place, in spite of the fact that the symposium programme starts with it, lies in the relation between observations and theory. 
Most of us would tend to call this a mainly theoretical symposium, but we should do well to consider what situation would exist if, for some unfortunate reason, all astrophysical and geophysical observations had been impossible. We then would have calculated by theory the possible existence of gas spheres in equilibrium like the sun; we might have a hunch of the solar corona but not of its beautiful streamers. In all probability, we would not even have guessed the existence of sunspots, nor of flares, nor of cosmic rays. Consequently this symposium would have been a good exercise of applied mathematics, illustrated by some laboratory experiments. It is only fair to say that, conversely, the absence of theoretical notions would have been even more disastrous: the observations then would have been an incoherent, nonsensical set of records.

This fiction story demonstrates that we do not really hope to grasp completely the complexity of the actual events in our theories. What we do hope is that our theories will help us to make sense of the observations, that is, to see which phenomena have a common cause, to distinguish certain chains of predictable events, and also to conceive of new observations, which may solve a crucial question in the interpretation of the data.

The theoretical investigations that are most fruitful deal with models. A model is a fictitious situation or experiment in which (unlike the real situation) all conditions are known. In discussing the real events, there may be many good reasons for giving a tentative or uncertain interpretation and for defending conflicting theories. In discussing model situations, however, there is no excuse for conflicting theories or obscure answers, for a precise question should (in the long run) receive a precise answer.

Just what are the model situations relevant to the topics of our symposium? In looking over the huge collection of papers that have appeared in the last seven years or so, I find it impossible to give even a brief summary of what has been accomplished $[3,4]$. But a brief classification of the problems, with some comments, may be useful (Table I).

Table I. Schematic classification of the theoretical problems

\begin{tabular}{|c|c|}
\hline $\begin{array}{c}\text { Fluid } \\
\text { 'Hydromagnetics' }\end{array}$ & $\begin{array}{c}\text { II } \\
\text { Ionized gas } \\
\text { 'Plasma dynamics' }\end{array}$ \\
\hline A. Basic equations & A. Basic equations \\
\hline $\begin{array}{l}\text { B. Problems with } \\
\text { external field }\end{array}$ & $\begin{array}{l}\text { B. Problems with } \\
\text { external field }\end{array}$ \\
\hline $\begin{array}{l}\text { G. } \text { Problems with } \\
\text { self-field }\end{array}$ & $\begin{array}{l}\text { G. } \text { Problems with } \\
\text { self-field }\end{array}$ \\
\hline
\end{tabular}


The fertile field of problems in column I has been opened by Alfvén with his studies of magneto-hydrodynamics, or, by a shorter term that has become quite popular, hydromagnetics. His main applications [5] were to astrophysical problems, where the conductor is an ionized gas, so that the term hydromagnetics might be assumed to include both columns I and II. However, the problems in column II have a longer standing. Appleton's magneto-ionic theory, if extended to lower frequencies to include the motion of the positive ions, is one of the central topics of пा B.

About five years ago the question whether the theories of I were applicable to the gases in II seemed a quite difficult one, especially as it was hard to tell what conductivity $\sigma$ to use: the full one along the magnetic lines of force, or the reduced one across the lines of force. By the work of Schlüter, Cowling, Piddington and others, this situation has now been cleared up. At the same time most authors have become more conscious of the various pitfalls. A theory is usually developed now either for I (fluids) or for II (ionized gas). This has caused a shift in the terminology: the term hydromagnetics (or magneto-hydrodynamics) is now very often reserved for column I.

Another important distinction is between the problems in lines B and C. In B an external magnetic field is given, the magnitude of which does not have to be questioned. If, moreover, it is supposed that the additional magnetic field caused by the induction currents in the investigated fluid or gas is smaller by an order of magnitude than the given field, the dynamic equations can be linearized. This was not needed in Alfvén's original presentation of the hydromagnetic waves, because of the particular orientation of the fields. But in all later extensions about waves with arbitrary polarization running through a medium in an arbitrary direction, the linearization is essential.

The problems in $\mathrm{c}$, in which both the character and the magnitude of the field are directly linked with the state of motion, are far more difficult. Hydromagnetic turbulence and the famous dynamo problem [6] belong to this class. They are basically non-linear and I shall not try a review. Certainly the problems in II are the hardest and have hardly been approached, in spite of their evident importance for the interstellar gas, stellar magnetic fields, etc. On the other hand the problems in I в are simplest; it is Dr Alfvén's merit to have found the right place to start the explorations. 


\section{STELLAR AND INTERSTELLAR FIELDS}

It should not be inferred from the introduction that the sun presents more exciting, or more important problems in the field of our symposium than astrophysics at large. In one respect, solar studies are unique: they show us so much detail that we lose at once our belief in simplified theories. But the sun is in all respects an average star, so that by sheer logic we may assume that anything we observe on the sun will appear in a more pronounced fashion in some other type of star.

Stellar magnetic fields have indeed been observed and offer a number of spectacular problems. Dr Babcock, the discoverer and almost exclusive author of the observations on this topic is present here, so there is no need for me to anticipate his lecture.

Interstellar fields have not so simple a history; perhaps I may relate a personal recollection. Oort and Burgers had before 1945 studied some problems in the aerodynamics of the interstellar gas. It seemed worthwhile to pursue these problems. In 1949 an international symposium on these matters was organized by I.A.U. and I.U.T.A.M. in Paris [7]. At that time magnetic fields had hardly been mentioned in this connexion and Oort was worried that only one or two participants would be able to judge their possible importance. It turned out, however, that almost everybody picked up this point and hydromagnetic turbulence formed one of the central topics of the discussions. At that time we were even unaware of work in the same directions that had started in other parts of the world.

The interstellar problems have continued to attract attention in connexion with the observable details of interstellar clouds, with the expansion of nova shells, and with the origin and acceleration of cosmic rays. Several conferences $[8,9]$ since that time have been devoted exclusively to one of these objects. The organizers of the present symposium have decided to place the emphasis on problems of the sun and the solar system and on stellar magnetism. Only a few papers remind us of the vast and interesting field outside these main topics.

\section{INTERPLANETARY SPACE}

Seven papers in the programme deal with problems presented by interplanetary space. Interest in this subject has sprung up recently from many sides. Before that time, we knew that the earth was at one astronomical unit from the sun with above it the ionosphere, which in practice could be studied only to the reflexion level in the $\mathrm{F}_{2}$ layer, say, at $250 \mathrm{~km}$. Theorists 
had ventured to about 1000 or $2000 \mathrm{~km}$ and there were also some ideas about a ring current at the order of an earth radius away from the surface, i.e. at a distance still less than o.0oor a.u. At the other end of the line we knew that the normal solar corona extended to roughly five solar radii, or $3,500,000 \mathrm{~km}=0^{\cdot 02}$ a.u. Occasional coronal streamers extended slightly further. For the rest, i.e. for $98 \%$ of the distance, interplanetary space seemed sufficiently empty not to worry about it. This has now changed. A list of the new lines from which evidence has come, or may still come, may be arranged as follows:

\section{Near the earth}

(a) Whistlers, by the current theory, present evidence of a density of the order of $10^{3}$ electrons $/ \mathrm{cm}^{3}$ about one earth radius above the surface.

(b) Observation of radio sources or moon echoes through the ionosphere at frequencies of the order of $\mathrm{I}^{-20} \mathrm{Mc} / \mathrm{s}$ has made it possible to obtain, in principle, some information about the entire ionosphere, including the top half.

(c) The suggestion has been made that the counterglow ('Gegenschein') of the zodiacal light should be explained by radiation from a gas tail of the earth, extending to about ten earth radii.

(d) Artificial satellites are now scheduled to go up to $500 \mathrm{~km}$ but may in a later stage of development give valuable information about the interplanetary medium.

\section{Near the sun}

(e) Red and infra-red eclipse photometry from an aircraft has shown the corona to extend to at least eighteen solar radii.

$(f)$ Radio observations of the Crab nebula at the time when the sun passes it have consistently shown that the radio waves are scattered in the outer corona. This effect becomes noticeable at twenty solar radii.

$(g)$ New attention has been given to theories of the escape of electrons and protons (evaporation) from the outer parts of the corona.

\section{Between sun and earth}

(h) Observations of comet tails indicate the action of corpuscular streams with densities of the order of $10^{3}$ particles $/ \mathrm{cm}^{3}$.

(i) Older measurements of the polarization of the zodiacal light have been confirmed. Unless extreme assumptions about the polarization of light scattered by interplanetary dust are made, these measurements prove the existence of 200 to 800 free electrons $/ \mathrm{cm}^{3}$ at one a.u. from the sun. 
(j) Observations of cosmic rays strongly indicate that the interplanetary medium affects the intensity and time of arrival both of the galactic cosmic rays and of the cosmic rays from solar flares.

It would be inadequate, in the present context, to attempt a more complete discussion. Reference may be made to various review articles [10, 11] and to the reports of the I.A.U. General Assembly at Dublin [12] for details. It would seem that the data provided by points $(h)$ and $(i)$ in the list are sufficiently certain as a basis of the discussion of the effects $(j)$, which will form a topic of discussion at this symposium.

\section{REFERENCES}

[1] Glerke, A. M. Problems in Astrophysics (London: A. and C. Black, 1903).

[2] Bullard, E. and others. Proc. Roy. Soc. A, 233, 289, 1955.

[3] Lundquist, S. Arkiv för Fysik, 5, $297,1952$.

[4] Spitzer, L. Physics of Fully Ionized Gases (Interscience Publishers, Inc., New York, 1956).

[5] Alfvén, H. Cosmical Electrodynamics (Oxford University Press, 1950).

[6] Elsasser, W. M. Rev. Mod. Phys. 28, 1 35, 1956.

[7] Problems of Cosmical Aerodynamics (ed. J. M. Burgers and H. C. van de Hulst), Proceedings Paris Symposium 1949 (Central Air Documents Office, Dayton, Ohio, 195 I).

[8] Gas Dynamics of Cosmic Clouds (ed. H. G. van de Hulst and J. M. Burgers), Cambridge Symposium 1953, I.A.U. Symposium Report Number 2 (North Holland Publishing Company, Amsterdam, 1955).

[9] Guanajuato conference on the origin of cosmic rays, September 1955; no report published.

[Io] van de Hulst, H. C. The Solar System (ed. G. P. Kuiper), vol. I, The Sun, Univ. Chicago Press, ch. 5, 'The Chromosphere and the Corona', 1953.

[II] van de Hulst, H. G. The Solar System (ed. G. P. Kuiper), vol. rv, Minor Planets, Meteorites, Comets and Origin of Solar System, ch. II, 'The zodiacal light' (in preparation).

[12] I.A.U. Transactions, vol. 9 (Dublin), (see reports of commissions 13, I5 and 22a), (Cambridge University Press, 1957).

\section{Discussion}

Biermann: I would like to make a remark concerning one special point of Dr van de Hulst's survey. The long time scale of the cycle of solar activity (twenty-two years) does not necessarily indicate that it should be regarded as directly connected with the cause of all the phenomena of shorter time scale. Since the subject will come up again on Thursday, I only wish to point out that there are reasons to believe that the turbulence of the hydrogen convection zone-which is to be regarded as deep for this purpose-is the main cause of most of the phenomena such as the solar activity. This was discussed at Dublin at the conference on turbulence in stellar atmospheres at some length (I.A.U. Transactions, vol. 9 (I957)). As I am going to explain in more detail on Thursday, meridional circulations should be present in the convective zone, the 
properties of which are connected with the structure of the turbulence. Its period is necessarily long compared with the characteristic time scale of turbulence elements even at larger depths.

van de Hulst: I look forward to the discussion on those points on Thursday.

Swann: One can think of several phenomena associated with a body of the size of a star and which have periods associated with that size. Thus, there are the periods of mechanical vibration, the period of electrical oscillation depending upon equivalent self-inductance and capacity and so on. I should like to ask Dr van de Hulst whether he can cite to us phenomena of these or analogous kinds which can have periods of the order of twenty-two years. Does my question make sense?

van de Hulst: The question makes sense and I hope someone will answer.

Gold: None of the phenomena the chairman mentioned has periods of the right order. The discussions I am aware of seemed to show that none other than some form of torsional magnetically coupled oscillations could be invoked. For such oscillations, however, the quantities involved can be rather arbitrarily chosen to fit the period.

Alfvén: Van de Hulst has remarked that a very important question is the density of interplanetary space. This topic will not be the subject of any particular paper during this symposium, but it will certainly be of importance to many of the problems to be considered here. I think it would be interesting to discuss it a little now although we shall probably come back to it many times later. Van de Hulst said that the value of the density is derived under the assumption that only half of the polarization is due to grains and the other half of the polarization is due to the electrons. Of course there are very seldom two independent effects just about equal so one may hesitate a little to accept this value.

Further, the value of the density from the whistlers seems not to be very definite. The whistlers, namely, are measured in a very low-frequency range and the theory which gives about the same density as that from polarization measurements is based on the assumption that only the motion of electrons is taken into account. But the whistlers occur at such low frequencies that it is possible that even ions interfere, and that would modify the values obtained. In this case there seems to be a possibility that we have a much lower value of density.

The emission of beams in the interplanetary space and quite a few other phenomena which we will discuss later indicate a much lower density. Different estimates point in the direction that a value of one, or even a lower value, would be in better agreement with these phenomena.

Dungey: I agree with Professor Alfvén on the importance of determining the interplanetary electron density from the zodiacal light. The whistler value refers to particles which are trapped by the earth's field and need not be the same as the interplanetary value. There should be a permanent geomagnetic effect due to the interplanetary ionized gas.

van de Hulst: I wish to answer this question about the zodiacal light. The ranges given in the paper were obtained with estimates of the dust polarization that I consider extreme. About 500-600 electrons $/ \mathrm{cm}^{3}$ are obtained if the dust 
polarization is zero. If it is positive, then the electron density gets lower; if it is negative the electron density gets higher. We can at most assume $15 \%$ dust polarization under these angles on the basis of theoretical computations. So I feel fairly sure that the range of estimates is correct. But if somebody assumes $25 \%$ instead of $15 \%$ dust polarization, then of course the lower limit may go down from 200 electrons $/ \mathrm{cm}^{3}$ to zero.*

Swann: If it should be trapping of electrons by an external magnetic field that is responsible for the somewhat higher values, we have a situation where there will be a rate of disappearance in that trapped region, by recombination, and a rate of supply, in which the balance determines the number present.

Ferraro: Like Professor Alfvén I should be happier if the electron density of the interplanetary gas were lower than the value of $200-800$ electrons $/ \mathrm{cm}^{3}$ suggested by van de Hulst. Corpuscular theories of magnetic storms indicate that the density of solar streams of corpuscles is of the order of I-Ioo electrons/ $\mathrm{cm}^{3}$. It is difficult to see how the streams could push their way through this denser gas.

van de Hulst: The data I have presented do not indicate that the gas should be stationary. It may be moving away from the sun continuously.

Ferraro: If the interplanetary gas were streaming towards the earth we should expect some sort of geomagnetic effect to be made manifest at the earth's surface.

Dungey: I think that you should certainly get the Chapman-Ferraro situation all the time; this is one possible happening. In such a situation the field is confined by the surrounding gas stream and is formed into a cavity surrounding the earth. Its dimensions can be estimated and depend on the electron density. The dimension of the cavity is proportional to the inverse one-sixth power of the density and with Siedentopf's analysis the radius of the cavity is about ten earth radii. If you put the density down by a factor of rooo you come up to thirty radii which is still quite a small thing.

Alfvén: If you eject a piece of matter from the sun and suppose that it moves radially outwards with constant velocity, then the density will decrease as $\mathrm{I} / r^{2}$. If you assume that this emission is just below the visibility in the corona, i.e. if you assume that the density at a distance of five solar radii just equals the electron density in the corona, then the value you get for the density in the beam at the earth's orbit is less than ro electrons $/ \mathrm{cm}^{3}$. For any assumption about the radial emission of beams which actually pass through the corona we cannot suggest a density inside the beam at the earth's orbit which is above this figure.

Simpson: The question of temperatures for the ionized gas in interplanetary space is also important for our understanding of the possible range of ion densities, which could exist in space. Chapman has recently calculated that, even at the distance of one a.u., the equivalent temperature may be remarkably high (about $10^{5}{ }^{\circ} \mathrm{K}$ ). Although we know very little experimentally about these temperatures, perhaps you would be willing to discuss this question and its relation to the expected range of gas densities in the interplanetary medium.

van de Hulst: In the outer corona we may speak of a temperature of the order

* 'Les particules solides dans les astres', Symposium Report, Mém. Soc. Roy. Sci. Liège, 15 (r955), H. C. van de Hulst, 'On the polarization of the zodiacal light', p. 89. 
of $10^{6}{ }^{\circ} \mathrm{K}$. But I do not agree with Ghapman's assumptions of a stationary gas with a well defined temperature. At one a.u. from the sun the mean free path is not small and it is difficult to talk about any temperature at all.

Parker: Chapman (Smithsonian Contr. to Astrophysics, 2, I, 1957), using the thermal conductivity of a tenuous ionized gas, and assuming that the interplanetary medium is in hydrostatic equilibrium in the solar gravitational field, has calculated that $n_{e}=500 / \mathrm{cm}^{3}$ and $T=2 \times 10^{5}{ }^{\circ} \mathrm{K}$ at the orbit of the earth. To attack his results one must probably argue that there are sufficiently strong interplanetary fields to reduce the thermal conductivity. 